Department of Cell Biology and Molecular Medicine

Faculty of Medicine and Faculty of Science and Informatics

University of Szeged, Hungary

\title{
MORPHOLOGICAL, IMMUNOCYTOCHEMICAL AND FUNCTIONAL CHARACTERIZATION OF THE MICROGLIAL PHENOTYPE IN CULTURE
}

Melinda Szabó

Summary of PhD Thesis

Thesis supervisor: Karoly Gulya, PhD, DSc

Szeged, 2015 


\section{Introduction}

Microglial cells, the resident immune cells of the central nervous system (CNS), share a number of phenotypic characteristics and lineage properties with other bone marrowderived myeloid cell populations, and are regarded as members of the monocyte/macrophage lineage. Microglia originate from primitive myeloid progenitors that arise early in embryonic development, and constitute an ontogenetically distinct population in the mononuclear phagocyte system. They are highly plastic and, by virtue of their location and current role in the nervous tissue, are able to undergo a variety of morphological and functional changes in response to various stimuli. Resting microglia display a ramified morphology characterized by numerous, fine-branched processes with relatively small somata and subdued macrophage-like functional properties. In response to neural injury, infection and inflammatory or other signals, microglia become activated and undergo a series of morphological, molecular and functional changes in proportion to the severity of the damage to the neuronal tissue. They become progressively less ramified and quickly develop an enlarged cell body with several short, thickened processes (activated microglia) that may eventually completely retract (phagocytic microglia). This coincides with their antigen presentation ability and cytotoxic and inflammation-mediating signalization.

As regards origin, there are two populations of microglial cells in the CNS at any given time. The resident microglia comprise a distinct pool of cells that respond to stimuli and proliferate accordingly, and regulate their population dynamics in a manner dependent on the severity of the tissue damage. They exhibit an extremely slow turnover with the bone marrow, lack the major histocompatibility complex (MHC) class I/MHC class II proteins, interferon- $\gamma$, cytokines, CD45 antigens and many other surface receptors required to serve in the antigen-presenting, phagocytic and cytotoxic roles characteristic of the normal macrophage functions. Another population of cells, however, among which the perivascular microglial cells are prominent in number, is located in the close vicinity of blood vessels, and can be replaced regularly by bone marrow-derived precursor cells that express MHC class II antigens, indicating their monocyte/macrophage origin. In cases of extreme damage to the CNS, as in infection or stroke, the blood-brain barrier may weaken considerably, and hematogenous, bone marrow-derived cells, such as myeloid progenitor cells and macrophages, may enter the brain. Once the damage has 
abated, the peripheral and central systems are disconnected for the recovery and regrowth period.

Similarly to the extensive studies on the origin and the morphological and functional development of the microglial phenotype in vivo, a large body of information is available on the characteristics of the different microglial populations maintained in cell cultures. However, as a variety of proinflammatory factors are produced by activated microglial cells on the one hand, and activation of microglial cells by a number of agents has been demonstrated on the other, characterization of the activated microglial cells in response to an in vitro stimulation/challenge predominates in the literature.

Microglial functions such as motility and phagocytosis are closely associated with dynamic changes in the cytoskeleton and related to intracellular calcium $\left(\mathrm{Ca}^{2+}\right)$ signaling. The ubiquitous $\mathrm{Ca}^{2+}$-binding proteins participate in $\mathrm{Ca}^{2+}$-elicited intracellular events, either as $\mathrm{Ca}^{2+}$-sensing/receptor/trigger or as $\mathrm{Ca}^{2+}$-buffering/transport proteins, by binding intracellularly stored $\mathrm{Ca}^{2+}$. They contribute to nearly all aspects of the functioning of the cell, and are important in numerous intracellular signaling processes, from the regulation of cellular homeostasis to learning and memory. Calmodulin $(\mathrm{CaM})$, one of the most important intracellular $\mathrm{Ca}^{2+}$ receptors, exerts its biological action through its heterogenous population of target proteins, which are involved in a number of cellular regulatory processes.

The nervous tissue is especially abundant in CaM. While its distribution has been characterized in detail for a number of neuronal cell types, its localization and functions in glial cells are much less known. Of all the glial components, only the microglia seem to have a considerable amount of CaM. They express a relatively large amount of CaM when activated (Casal ez al., 2001; Solá et al., 1997), and many aspects of their $\mathrm{Ca}^{2+}$ signaling are well documented (Färber and Kettelmann, 2006; Wong and Schlichter, 2014). CaM immunoreactivity or CaM gene-specific transcripts are often colocalized with those of the target enzymes of $\mathrm{CaM}$ within the same cytoplasmic compartments. For example, actin is accompanied by $\mathrm{CaM}$ in the cell cortex, helping to remodel the actinbased cytoskeleton in accordance with the actual (patho)physiological signals. Ionized calcium-binding adaptor molecule 1 (Iba1) is another intracellular $\mathrm{Ca}^{2+}$-binding protein with actin-binding capability that is expressed in macrophages and microglia, and is widely used to detect both resting and activated microglial phenotypes.

The modulatory action of $\mathrm{Ca}^{2+}$-bound $\mathrm{CaM}$ on multiple target proteins can be regulated by a number of compounds. Calmidazolium (CALMID; 1-[bis(4- 
chlorophenyl)methyl]-3-[2-(2,4-dichlorophenyl)-2-(2,4-dichlorobenzyloxy)ethyl]-1Himidazolium chloride) and trifluoperazine (TFP; 10-[3-(4-methylpiperazin-1-yl)propyl]2-trifluoromethyl-10H-phenothiazine dihydrochloride) are potent inhibitors of CaMrelated cellular activities. It is presumed that, apart from binding to the CaM protein, they can also exert their effects on some of the CaM-regulated targets directly.

\section{Specific aims}

Under physiological conditions, the vast majority of the resident microglia in the CNS are certainly unstimulated and characterized by ramified morphology. Despite the recent in vivo experimental approaches that unveiled new aspects of the functional, developmental and lineage characteristics of the microglial cell populations, only limited data are available on the development of the microglial phenotype in vitro under unstimulated/immunologically unchallenged conditions.

In an attempt to shed more light on the nature of the unstimulated microglia in vitro, we set out to characterize selected morphological, immunocytochemical and functional aspects of such cells, partially by quantitative techniques, in rat primary cortical cell cultures maintained routinely up to DIV28, in order to monitor the development of their phenotype. We analyzed the population dynamics of the microglial cells in terms of their percentage of the total number of cells during culturing, quantitatively characterized the different microglial populations according to their transformation indices, and differentially localized some of the canonical microglial markers to these distinct morphologies. As far as we are aware, a similarly detailed study for a period of up to 28 days on the characteristics of the in vitro development of unstimulated and unchallenged microglial cells of embryonic origin has not been reported previously.

The actin cytoskeleton is of paramount importance in many microglial functions. Its reorganization during cell migration, phagocytosis, or under several pathophysiological conditions, is in the forefront of research. One of the main regulators of the actin cytoskeleton reorganization is CaM. Relatively little is known, however, as concerns the possible involvement of CaM mediation in such important microglial functions as phagocytosis and the cellular functions associated with it, e.g. dynamic cytoskeletal reorganization. Thus, in view of the importance of CaM-mediated cell functions and the paucity of data on specific microglial functions related to and possibly 
regulated by $\mathrm{CaM}$, we set out to investigate the localization and intracellular distribution of $\mathrm{CaM}$ in pure microglial cell populations derived from rat primary mixed forebrain cultures by using immunocytochemical and Western blot techniques. Selected CaM inhibitors such as CALMID and TFP, previously reported to have different modes of action, were quantitatively tested for their ability to modify the microglial morphology (cell area, perimeter, transformation index), as well as lamellipodia, filipodia and podosome formation. Stimulation with LPS was used to evaluate the ability of microglial cells to respond to activation. Our specific aims were:

1) To determine the cellular composition and the rate of microglial proliferation in primary mixed cultures (DIV1-DIV28) prepared from rat embryonic forebrain tissues;

2) To quantitatively characterize the different microglial populations according to morphological parameters during culturing (DIV1-DIV28);

3) To localize some of the canonical microglial markers to these distinct morphologies during culturing (DIV1-DIV28);

4) To quantitatively characterize and localize microglial CaM immunoreactivities in relation to their morphologies in pure, secondary microglial cultures (subDIV4);

5) To characterize the effects of two CaM inhibitors (CALMID and TFP) on the morphological, including cytoskeletal, and immunocytochemical characteristics of pure microglial cultures (subDIV4).

\section{Materials and methods}

All animal experiments were carried out in strict compliance with the European Council Directive (86/609/EEC) and EC regulations (O.J. of EC No. L 358/1, 18/12/1986) regarding the care and use of laboratory animals for experimental procedures, and followed the relevant Hungarian and local legislation requirements. The experimental protocols were approved by the Institutional Animal Welfare Committee of the University of Szeged (I-74-II/2009/MÁB).

For a thorough characterization of different microglial phenotypes developed in vitro, we used various fluorescent immunohistochemical, -cytochemical, Western blot analytical, morphometric and functional (phagocytosis) studies, many of them quantitative, on 1) primary mixed neuronal/glial cultures prepared from embryonic (E18) rat forebrain tissues (DIV1-DIV28) and 2) pure secondary microglial cultures prepared 
from primary mixed cultures (subDIV4). Control (unchallenged) and LPS-challenged microglial cultures were compared with CaM inhibitors (CALMID and TFP) for several morphological, immunocytochemical and functional characteristics in primary mixed and pure secondary microglial cultures.

\section{Results}

Selected morphological, immunocytochemical and functional aspects of various microglial cell populations were characterized in mixed neuronal/glial and pure microglial cultures. The mixed primary cortical cultures were prepared from the forebrains of embryonic (E18) rats. Light microscopic fluorescent immunohistochemistry on E18 rat brains revealed that those of the the microglial cells that eventually gave rise to the cultures were mainly ameboid. The mixed cultures were maintained for up to 28 days (DIV1-DIV28). The pure microglial cells were subcloned (subDIV4) from the mixed primary cultures and maintained for up to 7 days (DIV7).

During culturing, expansion of the microglial cells was observed, as evidenced by quantitative assessment of selected monocyte/macro-phage/microglial cell-specific markers (HLA DP, DQ, DR, CD11b/c and Iba1) via immunocyto- and histochemistry and Western blot analysis. Fluorescent immunocytochemistry and Western analysis demonstrated a massive in vitro expansion of the microglia between DIV1 and DIV28. The Iba1 immunoreactivity in Western blots steadily increased about 750-fold, and the number of Iba1-immunoreactive cells rose at least 67-fold between DIV1 and DIV28. The number of microglia constantly increased from immediately after seeding (DIV1, 0.5\%) throughout the entire culturing period as the proportion of microglial cells reached $33.7 \%$ by DIV28.

Morphometric analysis on binary (digital) silhouettes of the microglia revealed their evolving morphology during culturing. Microglial cells were mainly ameboid in the early stages of in vitro differentiation, while mixed populations of ameboid and ramified cell morphologies were characteristic of older cultures as the average transformation index (TI, a dimensionless number that is an indicator for identification of the degree of morphological differentiation) increased from an average of 1.96 (DIV1) to 15.17 (DIV28). The ramified, resting microglia that developed later during the culturing could be characterized by a TI value as high as 81 . Throughout the experiments, microglial cells 
with TI $<3$ were considered ameboid. The ameboid, proliferating microglial cells, predominant in the early stages but always present throughout culturing, had TI values of less than 3, independently of the age of the culture, and were always Ki67-positive. The Ki67 immunoreactivity observed in the ameboid microglial nuclei usually exhibited a distinct dotlike pattern representative of the S/G2 phase unless it was associated with the periphery of the condensed chromosomes of the M-phase.

The microglial function was assessed by an in vitro phagocytosis assay. Phagocytosis was predominant in ameboid microglia. On DIV14, when mixed populations of ameboid and ramified microglia were present in the cultures in about equal numbers, microbeads were significantly more preferred by the ameboid forms (characterized by low TI values), while the ramified microglia (with higher TI values) were less active in phagocytosing microbeads. On average, microglia with TI $<3$ phagocytosed $11.08 \pm 8.6$ beads per cell (average \pm S.D., $n=39$ ), while microglia with $T I>3$ engulfed $3.16 \pm 3.6$ beads per cell $(n$ $=81)$; the difference was statistically significant $(\mathrm{p}<0.001)$.

Multiple immunofluorescence labeling of selected biomarkers revealed different microglial phenotypes during culturing. For example, while HLA DP, DQ, DR immunoreactivity was present exclusively in ameboid microglia $(\mathrm{TI}<3)$ between DIV1 and DIV10, CD11b/c- and Iba1-positive microglial cells were moderately (TI < 13) and progressively (TI < 81) more ramified, respectively, and always present throughout culturing. CD11b/c-positive microglia in cultures older than DIV10 were no longer able to express any of these HLA antigens.

Fluorescent immunocytochemistry and Western blot analysis demonstrated that a high concentration of $\mathrm{CaM}$ protein was characteristic of the mixed cultures throughout culturing. In young cultures (DIV1-DIV7), most of the CaM immunoreactivity was associated with non-microglial, e.g. mainly neuronal, cell forms. As the cultures aged, the CaM immunoreactivity localized to microglia became predominant. Moreover, CaM was differentially localized in ameboid and ramified microglia both in mixed and pure cultures. The strongest $\mathrm{CaM}$ immunoreactivity was always observed in the ameboid microglia, where the perinuclear area was the most intensely labeled. In ameboid microglia, the CaM and Iba1 immunoreactivities were distributed in a complementary manner, as the Iba1 protein tending to localize in the cell cortex and lamellipodia. The ramified microglia displayed an almost homogenous cytoplasmic Iba1 distribution with a considerably lower 
$\mathrm{CaM}$ content typically localized around the nucleus; the branches had only traces of CaM immunoreactivity.

CaM inhibition affected cell morphology and actin cytoskeleton reorganization. CALMID5 and CALMID50 resulted in increased area, perimeter and TI, whereas TFP alone strongly inhibited these characteristics. When challenged with lipopolisaccharide (LPS), the microglia became enlarged and acquired significantly larger perimeter and TI, consistent with these cells becoming activated. TFP sigificantly inhibited the expansion of cell surface area and perimeter both in unchallenged and LPS-challenged cells. In unchallenged and untreated (control) cultures, the Iba1 - and phalloidin-related fluorescence signals largely overlapped in the cell cortex of the mainly ameboid microglia, often in lamellipodia. Spot-like concentrations of phalloidin fluorescence resembling podosomes were often visible in LPS-treated cells. CALMID50 treatment resulted in phalloidin fluorescence that was clearly distributed in two distinct concentric rings in the cytoplasm, one ring in the cell cortex, and the other as a perinuclearly localized cytoplasmic streaming of freshly synthesized fibrous actin. Phalloidin-containing filipodia were also obvious in these cells. While the Iba1 immunoreactivity remained relatively intact, most of the phalloidin fluorescence intensity was lost in TFP-treated microglia indicating that TFP affected actin polymerization.

$\mathrm{CaM}$ inhibitors differentially altered the intracellular localization of $\mathrm{CaM}$, and affected the Iba1 and CaM protein expressions. Both unchallenged/untreated cells and LPS-challenged cells displayed high CaM content primarily localized in the perinuclear compartment and to a much lesser extent with that in the cell cortex. CALMID50 treatment often resulted in a cortically localized lamellipodia. After TFP10 treatment, the CaM immunoreactivity was very weak and homogenously distributed in the cytoplasm. CaM antagonists inhibited Ibal and CaM protein expressions with different efficacies as CALMID was less potent than TFP in affecting Iba1 and CaM protein expressions; both drugs showed dose-dependent inhibition.

\section{Summary of the findings}

In vitro studies on microglial population dynamics combined with phenotypic characterization can be of importance when different in vivo pathophysiological situations are modeled in vitro. Deciphering the roles of CaM in microglial functions, 
perhaps through use of different CaM-specific inhibitors, could be important in understanding the roles and modes of action of microglia in health and disease.

Our main findings were:

1) In primary mixed cultures, the number of microglial cells increased steadily. The Iba1 immunoreactivity in Western blots increased about 750-fold, and the number of Iba1-positive cells rose at least 67-fold between DIV1 and DIV28. The number of microglia increased from $0.5 \%$ to $33.7 \%$ during culturing.

2) In mixed primary cultures, TI values revealed the evolving microglial morphology and functions during culturing as microglia with $\mathrm{TI}<3$ were more active in phagocytosing than the ramified forms characterized with $\mathrm{TI}>3$.

3) The macrophage/microglia markers labelled different microglial phenotypes in culture: HLA DP, DQ, DR-positive microglia were exclusively ameboid, while CD11b/c-labeled microglia were typically less ramified than the Iba1-labeled cells. CD11b/c-positive microglia in cultures older than DIV10 were no longer able to express any of these HLA antigens.

4) As the cultures aged, the CaM immunoreactivity localized to microglia became predominant. CaM was differentially localized in ameboid and ramified microglia both in mixed and pure cultures. The stronges CaM positivity was found perinuclearly.

5) When CaM inhibitors were tested in unchallenged and in LPS-challenged pure microglia, they affected many morphological and functional aspects of microglial cells. Quantitative analysis showed that CALMID and TFP differentially affected the intracellular distributions of CaM and Iba1 and the actin cytoskeleton reorganization.

\section{Acknowledgements}

I thank Professor Karoly Gulya for his help. I am also grateful to my coworkers Miss Karolina Dulka, Mrs. Susan Ambrus and Mrs. Olga Daranyi. This work was supported by program project grants to the University of Szeged from the Ministry of National Resources (TÁMOP-4.2.1.B-09/1/KONV-2010-0005 and TÁMOP-4.2.2.A-11/1/KONV2012-0052) through the European Union Cohesion Fund. I dedicate this work to my family for their help and love. 


\section{List of publications directly related to the thesis:}

1) Szabo M, Gulya K (2013) Development of the microglial phenotype in culture. Neuroscience 241:280-295. (IF: 3.327)

2) Szabo M, Dulka K, Gulya K (2016) Calmodulin inhibition regulates morphological and functional changes related to the actin cytoskeleton in microglial cell cultures. Brain Research Bulletin 120:41-57. (IF: 2.718 - legfrissebb adat 2014re)

\section{Presentations (talks, posters) directly related to the thesis:}

1) Szabo M, Gulya K (2009) Glial cell population dynamics in vitro. Euroglia 2009.9th European Meeting on Glial Cells in Health and Disease Paris, France, 2009. Glia 57:S29-30.

2) Gulya K, Szabo M (2011) Development of the microglial phenotype in vitro. 8th IBRO World Congress of Neuroscience, Florence, Italy, 2011. D085.

3) Gulya K, Szabo M (2012) Calmodulin in cultured microglial cells. 8th FENS Forum of Neuroscience, Barcelona, Spain, 2012. A46-430 vol 7, p. 025.17.

4) Szabó M, Gulya K (2012) Development of the microglial phenotype in embryonic rat forebrain cultures. IBRO International Workshop, Szeged, Hungary 2012. Ideggyógy Sz 2012 65(S1):63.

5) Dulka K, Szabo M, Gulya K (2015) Calmodulin inhibition affects proliferation and cell viability in unchallenged and LPS-challenged pure microglial cultures. XII European Meeting on Glial Cells in Health and Disease, Bilbao, Spain, 2015. Glia 63:E95. 